\title{
Preliminary Benchmarking Efforts and MCNP Simulation Results for Homeland Security
}

\author{
Robert B. Hayes, PhD, CHP, PE \\ Senior Scientist \\ Remote Sensing Laboratory \\ Las Vegas, NV 89193 \\ hayesrb@nv.doe.gov
}

Keywords: Monte Carlo, Benchmark, Detector, Homeland Security, Testing

\section{Executive Summary}

It is shown in this work that basic measurements made from well defined source detector configurations can be readily converted in to benchmark quality results by which Monte Carlo N-Particle (MCNP) input stacks can be validated. Specifically, a recent measurement made in support of national security at the Nevada Test Site (NTS) is described with sufficient detail to be submitted to the American Nuclear Society's (ANS) Joint Benchmark Committee (JBC) for consideration as a radiation measurement benchmark. From this very basic measurement, MCNP input stacks are generated and validated both in predicted signal amplitude and spectral shape. Not modeled at this time are those perturbations from the more recent pulse height light (PHL) tally feature, although what spectral deviations are seen can be largely attributed to not including this small correction. The value of this work is as a proof-of-concept demonstration that with well documented historical testing can be converted into formal radiation measurement benchmarks. This effort would support virtual testing of algorithms and new detector configurations. 


\section{Introduction}

Prior to use for any significant application, proper operation of a system would be tested to verify minimal performance capability. Such is the case with detector design in terms of both software and hardware for homeland security. If detectors could be tested based on Monte Carlo models, then rigorous benchmarks should be generated to validate those models in order to make them reliable. Ideally, if all credible cargo configurations could be categorized in reasonably sized groupings such that measurement benchmarks could be made for the entire assortment of gamma spectrometers, these could serve to validate the Monte Carlo methods to test algorithms and aid in developing better algorithms.

The benchmarks would ideally have multiple source detector configurations with varying amounts of interstitial shielding. If the Monte Carlo methods were able to show for a given source detector configuration with multiple shielding layers that they could accurately interpolate the measured values for each shielding layer, it could safely be relied upon to extrapolate the effects of more or less shielding than was used in the benchmarks. The same applies to using different types of shielding, different sizes or numbers of detectors, and different volumetric source distributions.

The ANS JBC classifies a benchmark as a well defined and characterized radiation measurement that has been peer reviewed (ideally by the JBC). A more comprehensive description would be that if an independent scientist or engineer were to reproduce that benchmark configuration, they could literally quantify all dominant contributing physical differences between their own set up and that of the benchmark based on the benchmark write up. In addition, the measurement results must also be comparably described. The intended outcome could be restated that if said researcher, scientist, or engineer were to try and reproduce the measurement, all differences in the measurement results could again be fully quantified based on the measurement results presented in the benchmark write up. 
This paper is a proof of concept for this approach using MCNPX (Pelowitz 2005).

\section{Experimental Configuration}

The measurements were carried out with a commercial handheld detector system called an Identifinder (or Gr-135) made by Thermo-Eberline. The experimental configuration modeled is shown in Figure 1. The support for the source was not modeled as it was metal and not considered a dominant scattering source. The actual source stand was a plywood box made of 1" plywood which was modeled and is shown in Figure 1. The materials with the chemical compositions, dimensions, and densities modeled are all given in the Appendix as an MCNPX input stack for the Cf neutrons. The gamma detector is a right circular cylinder as is the neutron counter underneath it.

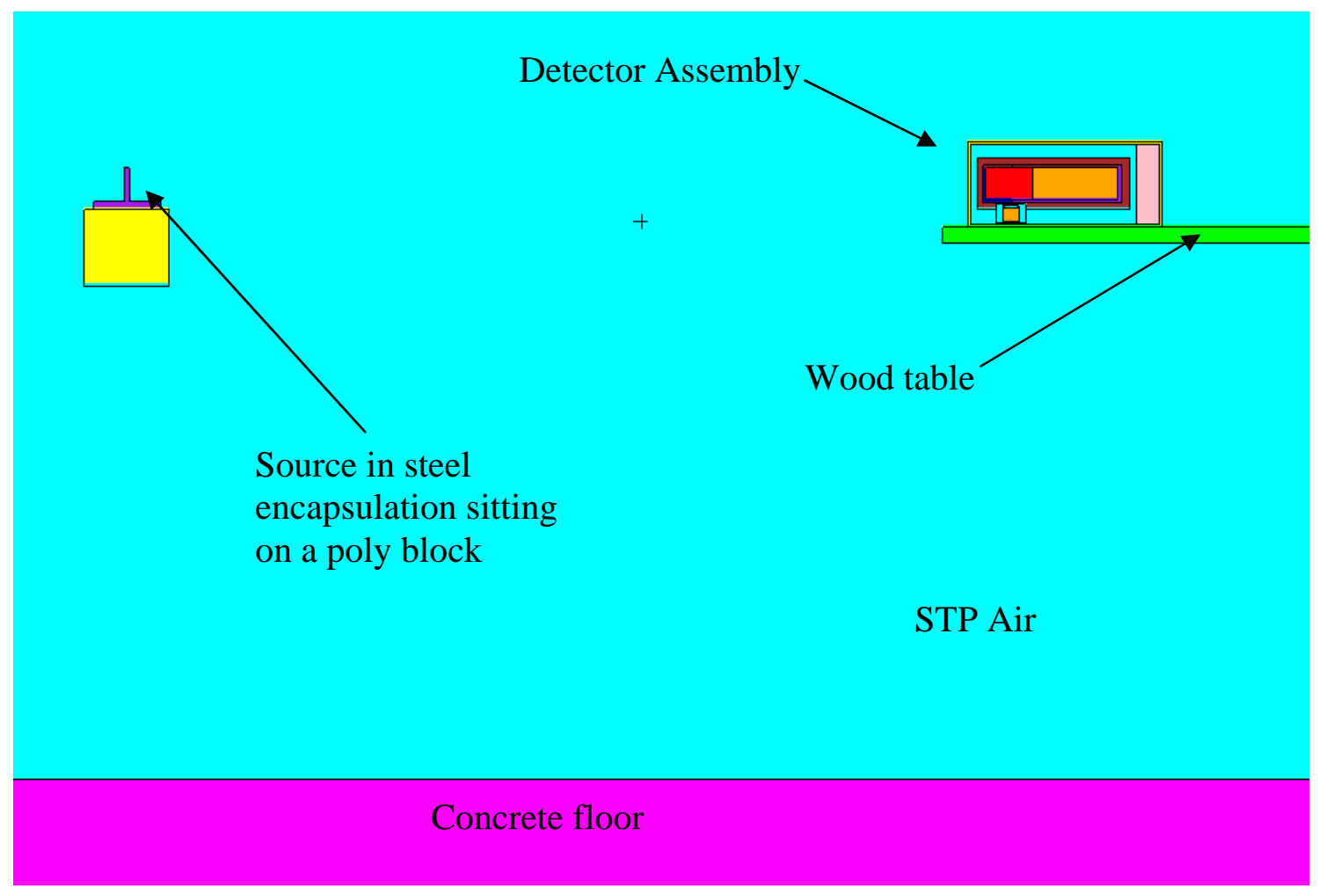

Figure 1. Modeled MCNP configuration for experimental setup. 


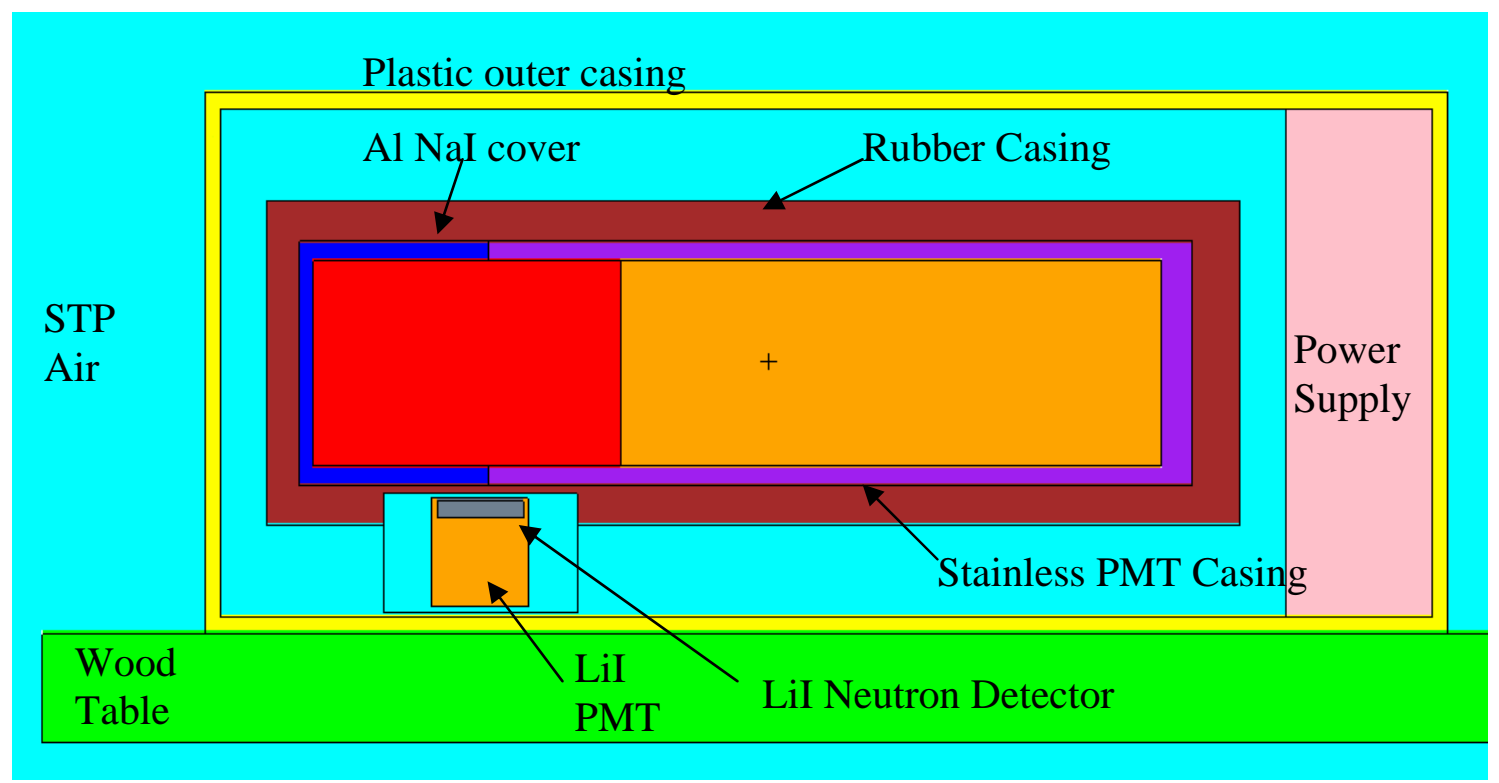

Figure 2. Close-up view of detail used for detector modeling. Engineering judgment was utilized to determine adequacy of electronics element modeling and peripheral interior infrastructure.

The neutron source spectrum was that of a Watt spectrum recommended in the MCNP manual for Cf-252 (Pelowitz 2005). The prompt and delayed gammas were taken from the approximations given by Shultis and Faw (2000) for U-235 fission using the corrections recommended by Valentine (1999) for Cf-252 spontaneous fission prompt gammas. The approximation for prompt gammas from U-235 fission is given in Equation 1 with the approximation for delayed gammas given in Equation 2.

$N_{\text {prompt }}(E)= \begin{cases}6.6 & 0.1<E<0.6 \mathrm{MeV} \\ 20.2 e^{-1.78 E} & 0.6<E<1.5 \mathrm{MeV} \\ 7.2 e^{-1.09 E} & 1.5<E<10.5 \mathrm{MeV}\end{cases}$

$N_{\text {delayed }}(E) \approx e^{-1.1 E}$

To adjust Equation (1) to approximate that of Cf-252 spontaneous fission prompt gamma spectra, the energy distribution approximation for U-235 prompt fission was reduced to match the value reported by Valentine (1999). According to Valentine (1999), the 
average gamma energy of $\mathrm{U}-235$ fission is $0.97 \pm 0.02 \mathrm{MeV}$, whereas that for Cf-252 is $0.87 \pm 0.02 \mathrm{MeV}$. This adjustment was done by using Equation (1) but reducing all the stated limits by $0.1 \mathrm{MeV}$. The formula for the energy distribution of delayed gammas (Equation 2) is characterized as already being very approximate by Shultis and Faw (2000) and so was not adjusted further. In calculating the number of source photons, the gamma and neutron multiplicities of Valentine (1999) were used. These were 7.98 and 3.757 as the average number of gammas and neutrons emitted in a Cf-252 spontaneous fission event. As a reference, the average number of neutrons emitted per fission event is reported by Shleien et al. (1998) as 3.7, and the average number of gammas per fission is reported by Reilly et al. (1991) as simply between 7 and 10. As these independent sources were all consistent, the Valentine values were used. The half life for Cf-252 used to decay correct the source activity was 2.645 years for a 4.6 month decay from the initial NIST calibration.

\section{Results}

The spectral distribution of the measured and simulated Cf-252 source overall agreed quite well as shown in Figure 3. There were also 61 neutron counts in the neutron detector element. Some spectral shape differences can be noted, although the overall count rate from the simulation overestimated that of the measured value by only $0.8 \%$. There is a spectral quality difference that can be seen in the figure between the measured spectrum and the sum of the individually simulated components (sum spectrum). At the energy range of about 200 to $300 \mathrm{keV}$, the simulated sum spectrum and the measured spectrum fully agree in shape and intensity. Below this range, the overall shapes appear

to agree, but the sum spectrum underestimates that of the measured spectrum. Above this range, the sum spectrum underestimates the measured spectrum although maintaining the correct overall shape. The overall effect compensates in the total count rate estimates. 


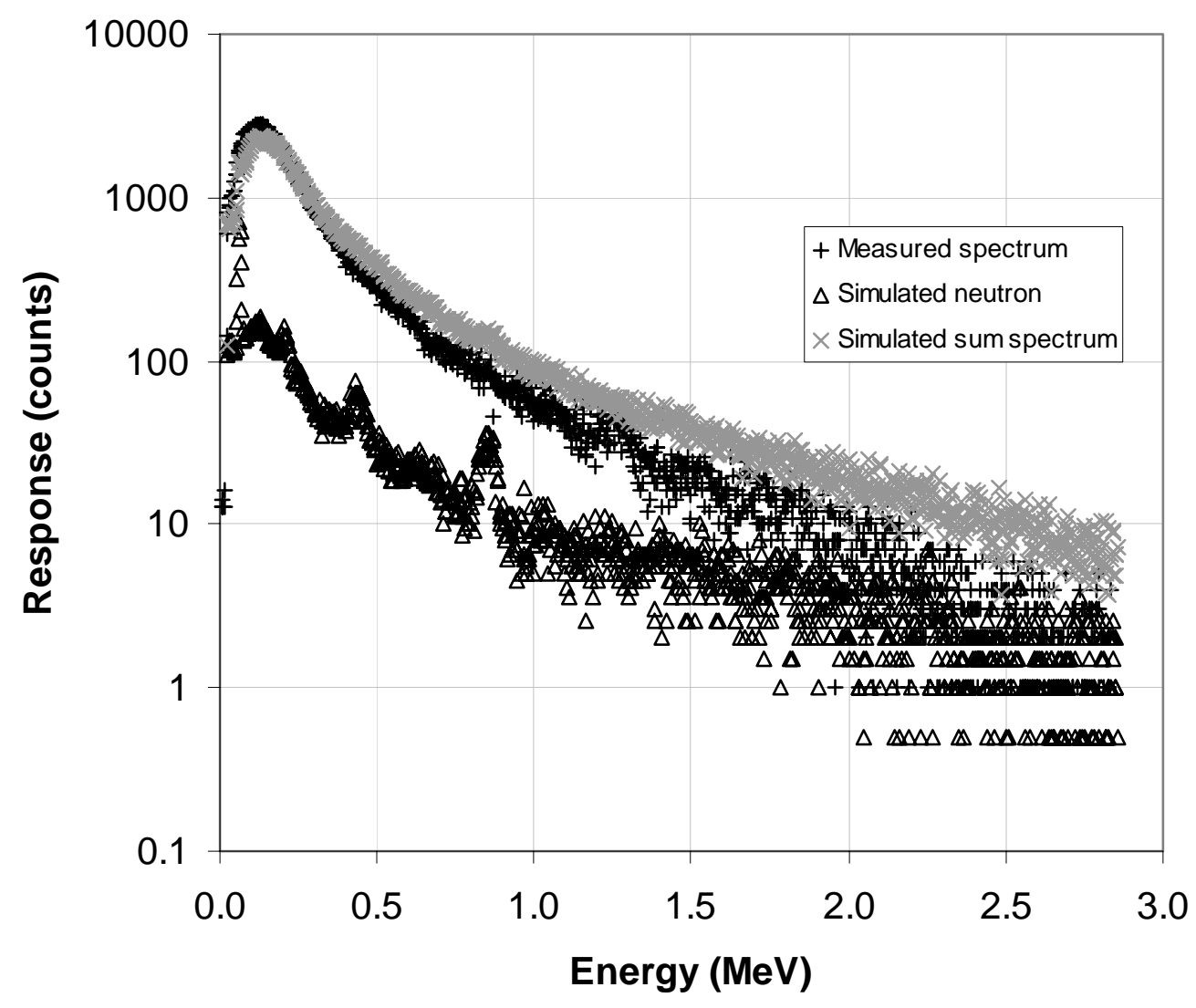

Figure 3. Simulated component of the measured spectrum and the actual measured spectrum in a semi log format.

The neutron count rate estimates in the LiI crystal require a knowledge of the lower energy threshold cutoff values obtained in a particular piece of instrumentation. For this reason, an energy distribution of the energy absorption events in the crystal was done to determine what value would be required to attain the same integrated count. These results are presented in Table 1 . What can be seen from Table 1 is that the apparent threshold neutron energy around $70 \mathrm{keV}$. As there were only 61 counts in the actual measurement with the LiI crystal, assuming Poisson statistics alone would put the error at 8 counts, placing the threshold energy between 70 and $80 \mathrm{keV}$ as the correct value to use for modeling purposes. 
Table 1. Neutron detector tally integrated count results using various neutron cutoff methodologies. The tally result \#1 utilized 100 shakes (a shake is1e-8 sec) and a minimum neutron energy of $10 \mathrm{keV}$ (if either limit is reached, the neutron is analog captured). The tally result \#2 used 1000 shakes and a $50 \mathrm{keV}$ neutron energy minimum.

\begin{tabular}{ccc}
\hline $\begin{array}{c}\text { Energy } \\
(\mathrm{keV})\end{array}$ & $\begin{array}{c}\text { Tally result } \\
\# 1\end{array}$ & $\begin{array}{c}\text { Tally result } \\
\# 2\end{array}$ \\
\hline 9 & 90 & 98 \\
18 & 87 & 95 \\
27 & 85 & 93 \\
36 & 82 & 91 \\
45 & 80 & 89 \\
55 & 79 & 83 \\
64 & 77 & 81 \\
73 & 61 & 65 \\
82 & 53 & 58 \\
91 & 51 & 54 \\
100 & 49 & 53 \\
1000 & 48 & 50 \\
2000 & 11 & 11 \\
\hline
\end{tabular}

\section{Discussion and Conclusion}

Given the lack of detailed tabulated gamma ray energies for both the prompt and delayed photons from Cf-252, the resulting simulated source values produced reasonable agreement with the measured result. The overall contribution to the NaI detector from the neutrons was small compared to that of the incident gammas. Both prompt and delayed gammas had an almost equivalent overall contribution to the detector response. Deviations in the spectral response for the NaI crystal can be attributed to lack of the PHL which, according to Heath (1964), would shift the spectrum to have higher counts below $0.5 \mathrm{MeV}$ and to have lower counts above this. This deviation would shift the spectrum simulated by MCNPX to be closer to that of the measured value.

The agreement between the measured and calculated values were better than was initially expected due the prerequisite assumptions of prompt and delayed gamma spectra approximations. This is likely attributable to the use of $\mathrm{NaI}$ as the detector with the inherent gamma smearing that takes place using this material. 
The success of this effort warrants further evaluations of historical testing results to eventually allow a complete suite of benchmarked data to be generated applicable to homeland security, operational health physics and research applications.

\section{References}

Erdtmann G, Soyka W. The Gamma Rays of the Radionuclides. Tables for Applied Gamma Ray Spectrometry. Verlad Chemie, New York, NY. 1979.

Heath RL. Scintillation Spectrometry Gamma-Ray Spectrum Catalogue. IDO-16880-1, Idaho National Engineering Laboratory, Idaho Falls, ID. 1964.

Pelowitz DB. MCNPX Users Manual. Version 2.5.0 April 2005. LA-CP-05-0369. Los Alamos National Laboratory, Los Alamos, NM. 2005.

Reilly D, Ensslin N, Smith H. Passive Nondestructive Assay of Nuclear Materials. NUREG/CR-5550, LA-UR-90-732. Los Alamos National Laboratory, Los Alamos, NM. 1991.

Shleien B, Slaback LA, Birky, BK. Handbook of Health Physics and Radiological Health $3^{\text {rd }}$ Edn. Lippincott Williams and Wilkins. New York, NY. 1998.

Shultis JK, Faw RE. Radiation Shielding. American Nuclear Society, La Grange Park, IL. 2000

Valentine TE. Evaluation of Prompt Gamma Rays for Use in Simulating Nuclear Safeguard Measurements. ORNL/TM/-1999/300. Oak Ridge National Laboratory. Oak Ridge, TN. Oct 1999.

This manuscript has been authored by National Security Technologies, LLC, under Contract No. DE-AC52-06NA25946 with the U.S. Department of Energy. The United States Government retains and the publisher, by accepting the article for publication, acknowledges that the United States Government retains a non-exclusive, paid-up, irrevocable, world-wide license to publish or reproduce the published form of this manuscript, or allow others to do so, for United States Government purposes. 


\section{Appendix}

\section{MCNPX Input Stack}

c Modeling Cf-252 measurement with handheld with NaI and LiI crystals

c

c outside universe

$\begin{array}{lll}1 & 0 & 1\end{array}$

c $\mathrm{NaI}$

$\begin{array}{llll}2 & 1 & -3.67 & -2\end{array}$

C $\mathrm{Al}$ case around detector

$\begin{array}{lllll}3 & 2 & -2.7 & -4 & 2\end{array}$

c interior of gr135 simulating preamp and pmt

$\begin{array}{llll}4 & 6 & -0.91 & -7\end{array}$

c power supply end of gr135

$\begin{array}{lllll}5 & 8 & -0.673 & -5 & 10\end{array}$

c interior air of gr135

$6 \quad 7 \quad-0.001205\left(\begin{array}{lll}-5 & -10 & 22\end{array}\right)(39: 38:-40)$

c plastic case of gr135

$\begin{array}{lllll}7 & 3 & -0.92 & 5 & -8\end{array}$

c air volume

$\begin{array}{llllllllll}8 & 7 & -0.001205 & 3 & 8 & -1 & 9 & 33 & 34 & 37\end{array}$

c celotex table

$9 \quad 4-0.8 \quad-9$

c concrete floor

$\begin{array}{lllll}10 & 5 & -2.35 & -3 & -1\end{array}$

c steel casing around pmt

$\begin{array}{llllll}11 & 9 & -7.82 & -30 & 7 & 2\end{array}$

c rubber detector assembly holder

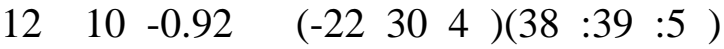

c steel source encasement

$\begin{array}{lllll}13 & 9 & -7.82 & -33 & :-34\end{array}$

c poly block source stand

$\begin{array}{llll}14 & 3 & -0.92 & -37\end{array}$

c air around LiI detector case

$15 \quad 7 \quad-0.001205\left(\begin{array}{lll}-38 & 40 & -39\end{array}\right)(41)$

c neutron detector pmt housing

$\begin{array}{llll}16 & 6 & -0.91 & (-41)(52)\end{array}$

c LiI crystal

$\begin{array}{llll}17 & 11 & -4.06 & -52\end{array}$

$1 \quad$ rCc $000-200 \quad 0001000500$

$\begin{array}{lllllllll}2 & \text { rCC } & 2 & 0 & 0 & 5.7 & 0 & 0 & 1.9\end{array}$

$\begin{array}{rrrrrrrrr}3 & \mathrm{pz} & -70 & & & & & & \\ 4 & \mathrm{rCC} & 1.75 & 0 & 0 & 3.5 & 0 & 0 & 2.25\end{array}$

$\begin{array}{llllllll}5 & \text { rpp } & 0.3 & 22.7 & -4.7 & 4.7 & -4.7 & 4.7\end{array}$ 


$\begin{array}{ccccccccc}7 & \text { rcc } & 7.7 & 0 & 0 & 10 & 0 & 0 & 1.9 \\ 8 & \text { rpp } & 0 & 23 & -5 & 5 & -5 & 5 & \\ 9 & \text { rpp } & -3 & 200 & -40 & 40 & -7 & -5 & \\ 10 & \text { px } & 20 & & & & & & \\ 22 & \text { rpp } & 1.15 & 19.15 & -3 & 3 & -3 & 3 & \\ 30 & \text { rcc } & 5.25 & 0 & 0 & 13 & 0 & 0 & 2.25 \\ 33 & \text { rcc } & -100 & 0 & -2 & 0 & 0 & 4 & 0.3 \\ 34 & \text { rpp } & -104 & -96 & -4 & 4 & -3 & -2 & \\ 37 & \text { rpp } & -105 & -95 & -5 & 5 & -12 & -3 & \\ 38 & \text { c/z } & 5.1 & 0 & 1.8 & & & & \\ 39 & \text { pz } & -2.4 & & & & & & \\ 40 & \text { pz } & -4.6 & & & & & & \\ 41 & \text { rcc } & 5.1 & 0 & -4.5 & 0 & 0 & 2 & 0.9 \\ 50 & \text { rcc } & 5.1 & 0 & -2.6 & 0 & 0 & 0.3 & 0.8 \\ 52 & \text { rcc } & 5.1 & 0 & -2.85 & 0 & 0 & 0.3 & 0.8\end{array}$

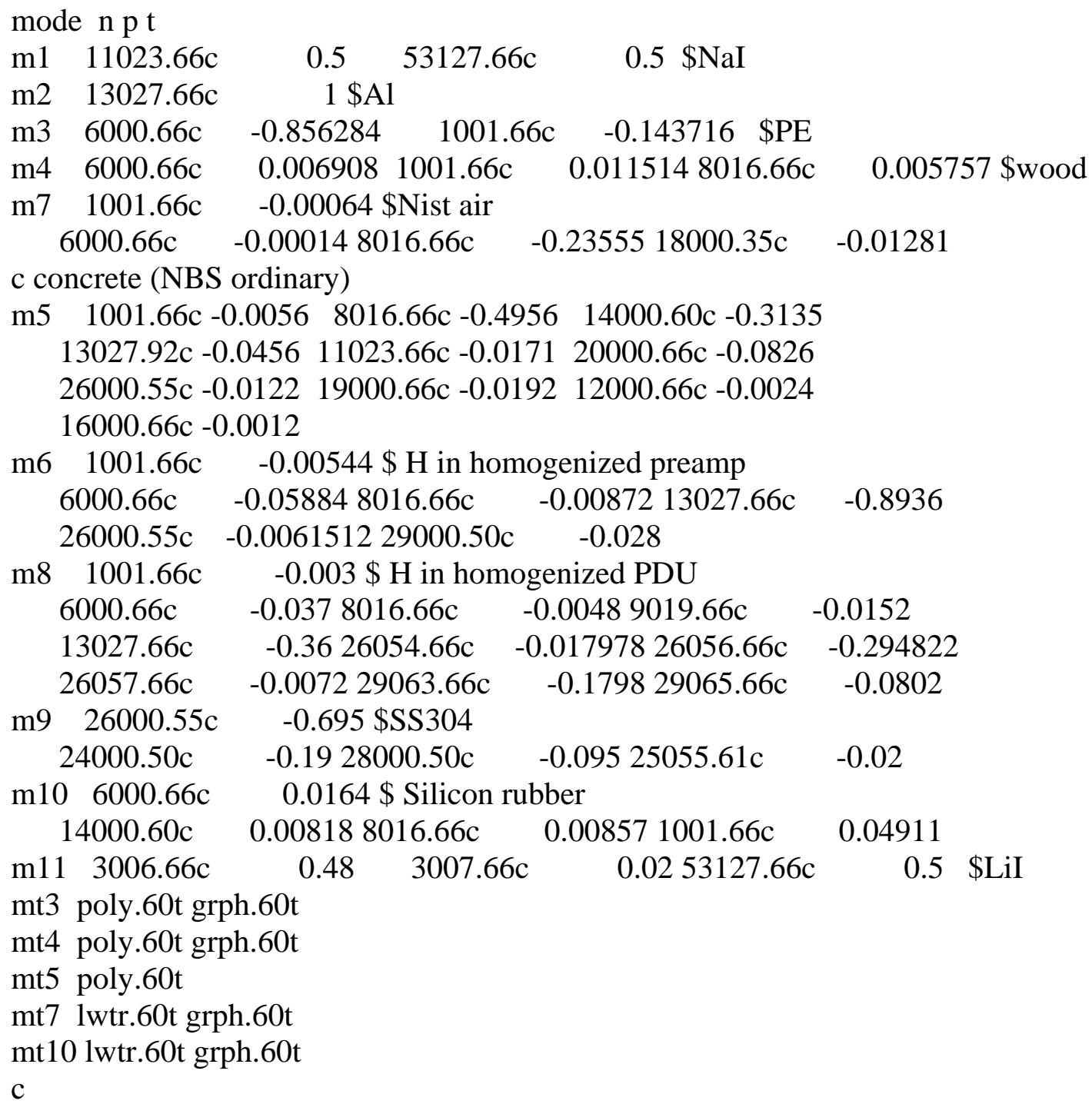




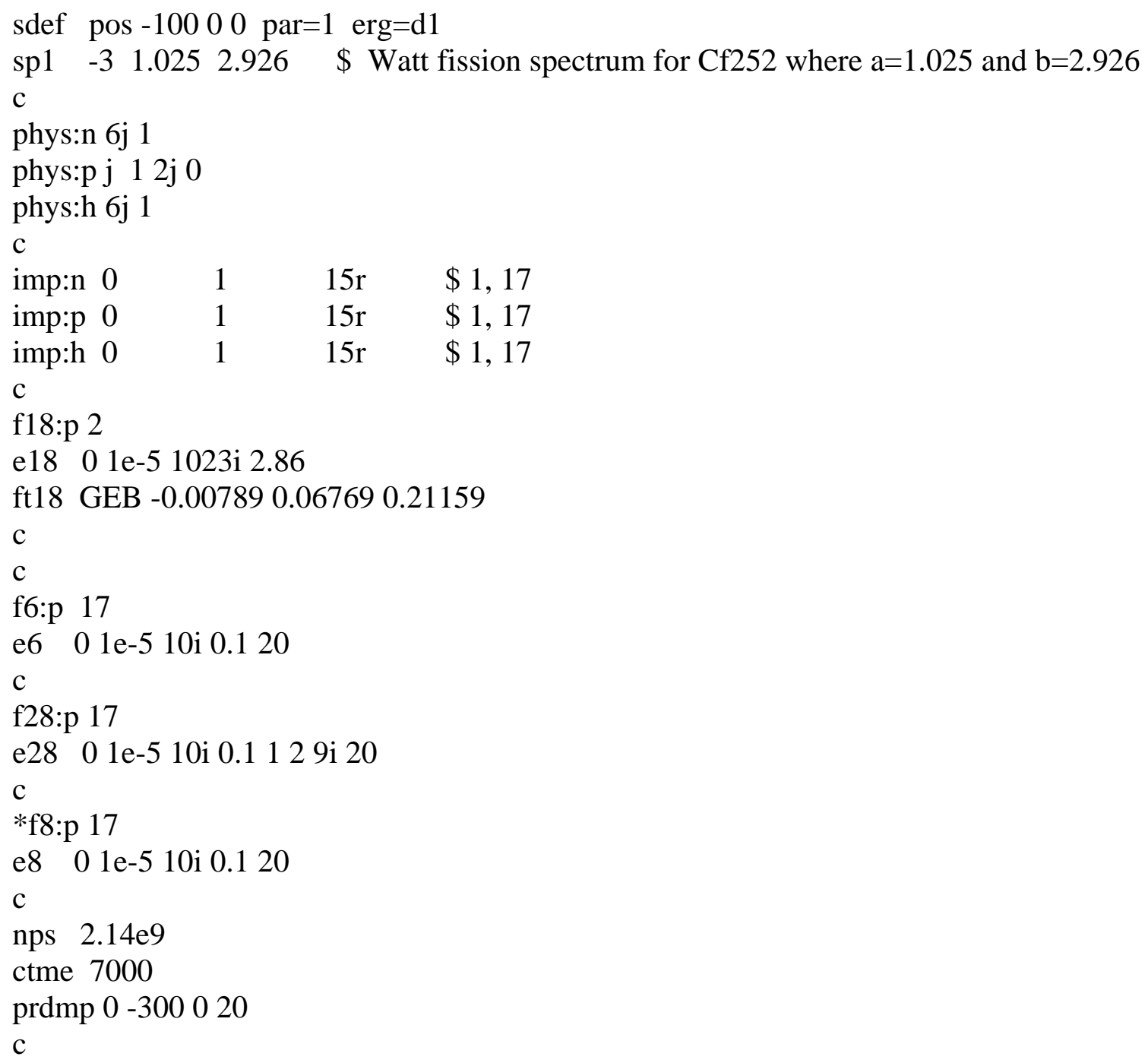

